\title{
Clarithromycin, ein neues Makrolid-Antibiotikum. Wirksamkeit bei puerperalen Infektionen und Übertritt in die Muttermilch
}

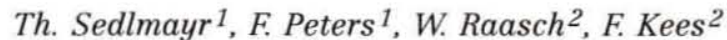 \\ ${ }^{1}$ St. Hildegardis-Krankenhaus Mainz \\ ${ }^{2}$ Lehrstuhl für Pharmakologie der Universität Regensburg
}

\section{Zusammenfassung}

Die vorliegende Studie untersucht die pharmakokinetischen und klinischen Parameter von Clarithromycin, einem neuen Makrolid-Antibiotikum, sowie seinen Übertritt in die Muttermilch. 12 Patientinnen (Alter von 24 bis 38 Jahren), die unter puerperalen Infektionen litten, wurden mit zweimal täglich $250 \mathrm{mg}$ Clarithromycin oral über 6 Tage behandelt. Blut- und Muttermilchproben wurden zu festgelegten Zeitpunkten entnommen. Mit Hilfe der HPLC und elektrochemischen Detektion wurden Clarithromycin und sein aktiver Metabolit, 14-Hydroxy-Clarithromycin, analysiert. Die Serumkonzentrationen von Clarithromycin fielen bei den untersuchten Patientinnen höher aus als die einer gesunden Kontrollgruppe (historischer Vergleich). Die höchsten Konzentrationen von Clarithromycin und 14-Hydroxy-Clarithromycin in der Muttermilch betrugen 25 bzw. $75 \%$ der entsprechenden Serumkonzentrationen. Alle Patientinnen wurden innerhalb von 2 bis 4 Tagen beschwerdefrei. Es waren keine Nebenwirkungen der Therapie erkennbar. Clarithromycin erscheint als ein geeignetes Antibiotikum zur Behandlung puerperaler Infektionen und wegen seiner beträchtlichen Konzentrationen in der Muttermilch insbesondere auch zur Behandlung der puerperalen Mastitis.

\section{Clarithromycin: Pharmacokinetics, Clinical Effectiveness Transmission into Breast Milk of a New Macrolide Antibiotic in Patients with Puerperal Infections}

The aims of this study were, to determine the pharmacokinetic parameters of clarithromycin and to study the passage of the drug into breast milk. Twelve patients (age 24 to 38 ; weight 44 to $83 \mathrm{~kg}$ ), suffering from puerperal infections, were treated orally with 250 mg clarithromycin b.i.d. for 6 days. Samples of blood and breast milk were taken at timed intervals. The specimens were assayed for clarithromycin and its active metabolite 14-hydroxy-clarithromycin by HPLC and electrochemical detection. Serum concentrations of clarithromycin in the investigated patients were higher than those reported in healthy volunteers. The mean peak concentrations of clarithromycin and 14-hydroxy-clarithromycin in breast milk were about $25 \%$, and $75 \%$ respectively of the corresponding serum concentrations. All patients recovered within 2 to 4 days and no drugassociated side effects (eg. gastrointestinal) were noted. Clarithromycin appears to be an appropriate antibiotic for the treatment of puerperal infections and (because of its considerable concentrations in breast milk) for puerperal mastitis as well.

\section{Einleitung}

Clarithromycin ist ein neues Makrolid-Antibiotikum mit einem ähnlichen Wirkspektrum wie Erythromycin, aber mit einem günstigeren pharmakokinetischen Profil. Es unterscheidet sich von Erythromycin nur durch eine Methoxy-Gruppe in Position C-6 anstatt einer Hydroxyl-Gruppe, welche Clarithromycin gegen den sauren $\mathrm{pH}$ des Magens stabilisiert (1).

Die Pharmakokinetik von oral verabreichtem Clarithromycin ist nicht-linear und komplex. Clari-

Geburtsh. u. Frauenheilk. 53 (1993) 488-491

(c) Georg Thieme Verlag Stuttgart · New York thromycin wird in vivo teilweise zum mikrobiologisch aktiven Metaboliten 14-Hydroxy-Clarithromycin reduziert (2). Untersuchungen im Bronchialsystem zeigten, da $\beta$ Wirkungssubstanz und Metabolit besonders hohe Gewebespiegel erreichten, die teilweise über dem Zehnfachen der Plasmaspiegel lagen; für die intrazelluläre Aufnahme wird dabei ein Carrier-vermittelter Transportmechanismus angenommen (3). Die durchschnittliche maximale Plasmakonzentration im steady state beträgt bei oraler Aufnahme von zweimal täglich $250 \mathrm{mg}$ Clarithromycin $1,0 \mu \mathrm{g} / \mathrm{ml}$ für die Muttersubstanz und $0,6 \mu \mathrm{g} / \mathrm{ml}$ für den aktiven Metaboliten (2). Die Halbwertszeit der Muttersubstanz in dieser Dosierung beträgt annähernd 3,5 Stunden und für den Metaboliten 4,7 Stunden. Diese Pharmakokinetik ermöglicht es, mit nur zwei Anwendungen pro 24 Stunden auszukommen. 


\begin{tabular}{|c|c|c|c|c|c|}
\hline Nr. & Pat: & Alter & Parität & Diagnose & Keim \\
\hline 1 & MA & 38 & III & Endomyometritis $n$. Sectio & Staph. aureus \\
\hline 2 & PE & 25 & 1 & $\begin{array}{l}\text { Endomyometritis } n \text {. Sectio } \\
\text { Bauchdeckenhämatom }\end{array}$ & $\begin{array}{l}\text { Mycoplasma hominis } \\
\text { (Lochien) }\end{array}$ \\
\hline 3 & RS & 33 & 1 & Sekundärnaht Episiotomie & E. coli \\
\hline 4 & $\mathrm{CL}$ & 25 & 1 & Endomyometritis post partum & $\begin{array}{l}\text { Ureaplasma urealyticum } \\
\text { Leptotrichia vaginalis }\end{array}$ \\
\hline 5 & RA & 28 & 1 & Harnwegsinfekt & Staph. aureus \\
\hline 6 & YA & 24 & 1 & Endomyometritis n. Sectio & Enterokokken \\
\hline 7 & LE & 28 & 1 & Endomyometritis n. Sectio & Streptokokken B \\
\hline 8 & wo & 27 & ॥ & Sekundärnaht Episiotomie & Staph. aureus \\
\hline 9 & $\mathrm{KL}$ & 29 & 1 & $\begin{array}{l}\text { Endomyometritis } n \text {. Sectio } \\
\text { Bauchdeckenhämatom }\end{array}$ & Enterokokken \\
\hline 10 & TR & 26 & 1 & Wundinfektion n. Sectio & Staph. aureus \\
\hline 11 & WE & 25 & 1 & $\begin{array}{l}\text { Sekundärheilung Episiotomie/ } \\
\text { Dammriß III. Grades }\end{array}$ & E. coli \\
\hline 12 & SI & 32 & & Sekundärnaht einer Episiotomie & Enterokokken \\
\hline
\end{tabular}

Tab. 1 Demographische Daten der Patientinnen und Diagnose.
Bei Infektionen in Gynäkologie und Geburtshilfe haben wir es in der Regel mit einer überschaubaren Verteilung von Keimen zu tun. Das Keimspektrum puerperaler Infektionen (Endomyometritis, Mastitis, Wundinfektion, Harnwegsinfekt) umfaßt in erster Linie Streptokokken A und B, Staph. aureus, E. coli, Enterokokken und Chlamydien. Bis auf die Enterobacteriaceae deckt Clarithromycin dieses Spektrum ab. Ein weiterer Anwendungsbereich liegt in der Therapie von Infektionen mit Mycoplasmen und Chlamydien. Ziel der vorliegenden Studie war es, die pharmakokinetischen Parameter von Clarithromycin bei Patientinnen mit puerperalen Infektionen $\mathrm{zu}$ bestimmen und den Übertritt des Medikamentes in die Muttermilch zu untersuchen.

\section{Patientinnen und Methoden}

Zwölf Wöchnerinnen im Alter von 24 bis 38 Jahren (Median 27,5 Jahre), Gewicht 44 bis $83 \mathrm{~kg}$ (Median 64 $\mathrm{kg}$ ), Größe 158 bis $179 \mathrm{~cm}$ (Median $168 \mathrm{~cm}$ ) wurden in die Studie aufgenommen. Sechs Patientinnen hatten eine Endomyometritis (Tag 4-8 post partum, Median 5,3), drei Patientinnen wurden anläßlich einer Sekundärnaht der Episiotomiewunde prophylaktisch behandelt. Eine Patientin litt an einer Pyelonephritis, zwei Patientinnen hatten infizierte Wunden (Pfannenstielschnitt und Episiotomie). Es wurden folgende Keime isoliert: $4 \times$ Staph. aureus, $3 \times$ Enterokokken, $2 \times \mathrm{E}$ coli, $1 \times$ Streptokokken $\mathrm{B}, 1 \times \mathrm{My}-$ coplasma hominis, $1 \times$ Ureaplasma urealyticum in Kombination mit Leptotrichia vaginalis (Tab. 1).

Alle Patientinnen wurden entsprechend der Helsinki-Charta über die Studie und das Medikament aufgeklärt und gaben ihr schriftliches Einverständnis. Sie wurden mit $250 \mathrm{mg}$ Clarithromycin in Kapselform zweimal pro die über sechs Tage behandelt. Die Substanz wurde uns freundlicherweise von der Fa. Abott GmbH, Wiesbaden, zur Verfügung gestellt. Während der Behandlung wurden die Kinder nicht gestillt; die Muttermilch wurde abgepumpt. Zur Bestimmung der Serum- und Muttermilchkonzentrationen von Clarithromycin und seines Metaboliten wurden zu folgenden Zeiten Proben genommen: Drei Tage lang jeweils morgens und abends im 12-Stunden-Abstand, nach Erreichen eines steady-state am vierten Tag, morgens vor Medikamentengabe, sowie 2, 4, 8 und 12 Stunden danach. Die Proben wurden bis zur Analyse bei $-30^{\circ} \mathrm{C}$ aufbewahrt.

Clarithromycin und sein aktiver Metabolit, 14Hydroxy-Clarithromycin, wurden mit Hilfe der Hochdruckflüssig- keitschromatographie (HPLC) und elektronischer Detektion bestimmt. Die untere Nachweisgrenze lag bei 40 bis $50 \mathrm{ng} / \mathrm{ml}$. Zusätzlich wurde die gesamte antibakterielle Aktivität mit Hilfe des Bioassays bestimmt (Abbott Laboratories, Chicago, Illinois, USA) (3). Die pharmakokinetischen Parameter wurden modellunabhängig aus den Serumkonzentration von Clarithromycin und 14-Hydroxy-Clarithromycin am vierten Therapietag berechnet.

\section{Ergebnisse}

\section{Klinik}

Das klinische Ziel der Entfieberung, Beschwerdefreiheit und/oder Wundheilung wurde bei allen Patientinnen erreicht. Die Einzeldaten der Diagnosen sind in Tabelle 1 angegeben. Alle Patientinnen schlossen die Behandlung, wie vorgesehen, ab. Es waren keine unerwünschten Wirkungen zu verzeichnen, insbesondere keine gastro-intestinalen Nebenwirkungen.

\section{Pharmakokinetik}

In Abb. 1 sind die medianen Konzentrationen von Clarithromycin und seines aktiven Metaboliten,

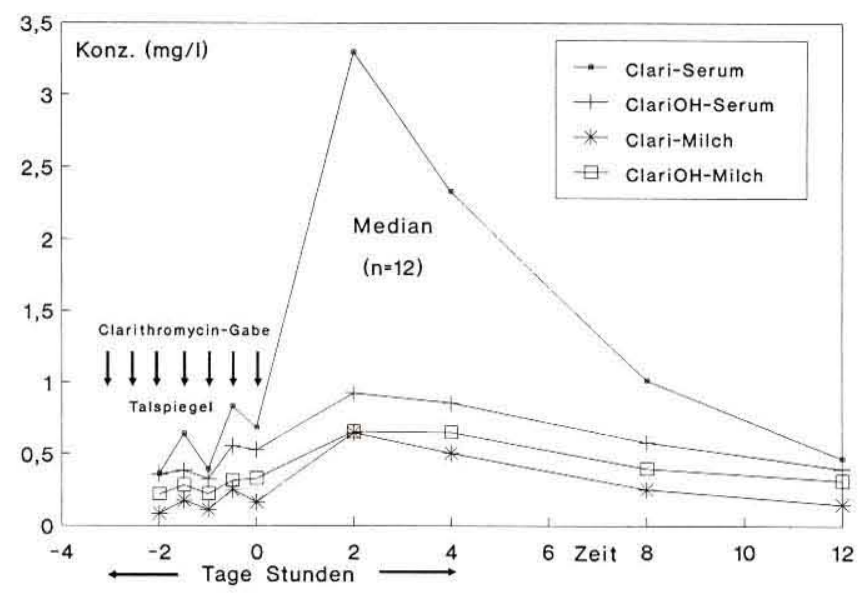

Abb. 1 Konzentrationen (Median) von Clarithromycin (Clari) und seines aktiven Metaboliten, 14-Hydroxy-Clarithromycin ( $\mathrm{ClaOH})$, in Serum und Milch von Wöchnerinnen $(n=12)$, gemessen im steady state nach dreitägiger Therapie mit $2 \times 250 \mathrm{mg}$ Clarithromycin pro Tag. 


\begin{tabular}{|c|c|c|c|c|}
\hline & \multicolumn{2}{|c|}{ Serum } & \multicolumn{2}{|c|}{ Muttermilch } \\
\hline & Clari & $\mathrm{ClaOH}$ & Clari & $\mathrm{ClaOH}$ \\
\hline $\mathrm{Cmin}(\mathrm{mg} / \mathrm{l})$ & $0,90 \pm 0,25$ & $0,59 \pm 0,10$ & $0,21 \pm 0,05$ & $0,36 \pm 0,05$ \\
\hline $\mathrm{Cmax}(\mathrm{mg} / \mathrm{l})$ & $3,59 \pm 0,64$ & $0,98 \pm 0,28$ & $0,85 \pm 0,12$ & $0,63 \pm 0,08$ \\
\hline $\mathrm{t} 1 / 2(\mathrm{~h})$ & $4,0 \pm 0,3$ & $8,6 \pm 0,6$ & $4,3 \pm 0,3$ & $9,0 \pm 1,2^{\circ}$ \\
\hline $\operatorname{tmax}(\mathrm{h})$ & $2,0 \pm 0,0$ & $2,7 \pm 0,3$ & $2,2 \pm 0,2$ & $2,8 \pm 0,3$ \\
\hline AUCO-12h (mg/l'h) & $21,0 \pm 3,8$ & $8,48 \pm 1,12$ & $5,29 \pm 0,72$ & $5,57 \pm 0,65$ \\
\hline
\end{tabular}

$\cdot n=9$ (SU)

Clari = Clarithromycin, $\mathrm{ClaOH}=14-\mathrm{Hydroxy}-\mathrm{Clarithromycin} ; \mathrm{Cmin}=$ Talspiegel unmittelbar vor der letzten Gabe, $\mathrm{Cmax}=$ maximale Konzentration, tmax $=$ Zeitpunkt der maximalen Konzentrationen; $t 1 / 2$ = Eliminationshalbwertszeit, AUCO-12h = Fläche unter der Kurve als MaB für die Bioverfügbarkeit, gemessen im steady State.

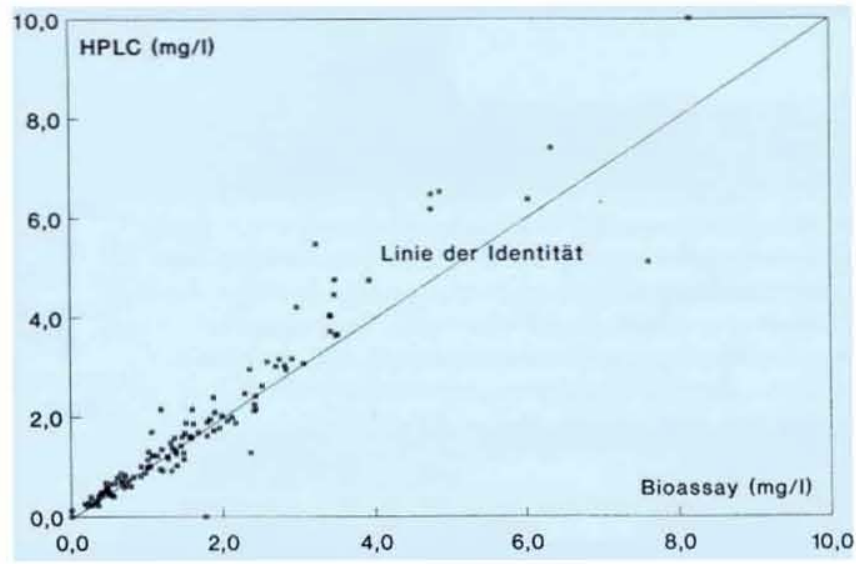

Abb. 2 Korrelation aus der Summe der Serumkonzentrationen von Clarithromycin (Clari) und seines aktiven Metaboliten, 14-HydroxyClarithromycin $(\mathrm{ClaOH})$, gemessen mit Hilfe der HPLC (Hochdruckflüssigkeitschromatographie) und der antibakteriellen Aktivität, gemessen mit dem Bioassay.

14-Hydroxy-Clarithromycin, in Serum und Muttermilch abgebildet, in Tab. 2 die berechneten pharmakokinetischen Parameter. Die steady-state-Serumkonzentrationen von Clarithromycin und seines wirksamen Metaboliten, 14-Hydroxy-Clarithromycin, waren mit den Serumkonzentrationen von mit zweimal täglich $500 \mathrm{mg}$ Clarithromycin behandelten gesunden Probanden vergleichbar (5). Die Konzentrationen des Metaboliten betrugen im Plasma ca. $25 \%$ und in der Muttermilch etwa $75 \%$ der Konzentrationen der Muttersubstanz. Sie zeigten einen flacheren Konzentrations-Zeit-Verlauf (Abb. 1). Eine gute Korrelation wurde für die Summe aus den Konzentrationen von Clarithromycin und 14-Hydroxy-Clarithromycin und der gesamten antibakteriellen Aktivität gefunden $(r=0,95$ in Serum, Abb. 2, bzw. $r=0,94$ in Muttermilch).

\section{Diskussion}

Die vorliegenden Ergebnisse belegen die klinische Wirksamkeit von Clarithromycin bei puerperalen Infektionen. Der Wirkstoff hat gegenüber Erythromycin günstigere pharmakokinetische Eigenschaften: bessere Resorption, höhere Serum- und Gewebespiegel, relative Stabilität gegen Magensäure und das Vorliegen des mikrobiologisch aktiven Metaboliten 14-OH-6-O-MethylErythromycin, der mindestens so wirksam ist wie Ery-
Tab. 2 Pharmakokinetische Parameter $(\mathrm{m} \pm$ SEM) von Clarithromycin und 14-Hydroxy-Clarithromycin in Serum und Milch von Wöchnerinnen $(n=12)$, gemessen im steady State nach dreităgiger Therapie mit $2 \times 250 \mathrm{mg}$ Clarithromycin pro Tag. thromycin. Die Verträglichkeit von Clarithromycin ist gut. Die unter Erythromycin bekannten Nebenwirkungen, wie Übelkeit oder Erbrechen, wurden in dieser Studie nicht beobachtet. Das Spektrum der häufigsten Infektionen im Wochenbett kann mit Clarithromycin abgedeckt werden. Die klinische Wirksamkeit bei E. coli-Infekten dieser Studie kann u.U. damit erklärt werden, daß andere bei der Keimisolierung nicht zutage getretene Erreger einer Mischbesiedelung die eigentlichen Auslöser der Erkrankung waren.

In der Muttermilch wurden beträchtliche Konzentrationen von Clarithromycin nachgewiesen. Die relative Menge des polaren Metaboliten, 14-Hydroxy-Clarithromicin, war, verglichen mit der Muttersubstanz, in der Muttermilch höher als im Serum, was Muttermilch als ein Ausscheidungskompartiment charakterisiert. Gestillte Kinder sollten deshalb für die Zeit der Behandlung mit Clarithromycin die Muttermilch nicht erhalten, um eventuellen Nebenwirkungen vorzubeugen.

Andererseits erscheinen die Eigenschaften dieses Antibiotikums für die Behandlung der Brustentzündung im Wochenbett besonders geeignet. Die puerperale Mastitis wird in über $90 \%$ der Fälle von Staphylococcus aureus verursacht (6). Zur adäquaten Therapie bieten sich heute beispielsweise ein entsprechend wirksames Cephalosporin oder Dicloxacillin an. Beide Substanzgruppen treten aber nur in sehr geringen Mengen in die Muttermilch über. Man würde deshalb eine Substanz mit bakteriziden Konzentrationen in der Muttermilch bevorzugen. Diese Eigenschaften trifft man beim Clarithromycin an. Deshalb erscheint die Substanz zur Behandlung der puerperalen Mastitis gut geeignet.

Zusammenfassend kann festgestellt werden, daß Clarithromycin in einer Dosierung von täglich $2 \times 250 \mathrm{mg}$ in oraler Form ein gut verträgliches und wirksames Antibiotikum zur Behandlung puerperaler Infektionen ist. 


\section{Literatur}

1 Morimoto, S., Y. Takahashi, Y. Watanabe, S. Omaru: Chemical modification of erythromycins. I. Synthesis and antibacterial activity of 6-0-methylerythromycins. J. Antibiot. 37 (1984) 187 -189 .

2 Ferrero, J. L., B. A. Bopp, K. C. Marsh, S. C. Quigley, M. J. Johnson, D. J. Anderson, J. E. Lamm, K. G. Tolmann, S. W. Sanders, J. H. Cavanaugh, R. C. Sonders: Metabolism and disposition of clarithromycin in man. Drug. Metab. Disp. 18 (1990) 441-446.

3 Kohno, Y., H. Yoshida, T. Suwa, T. Suga: Uptake of clarithromycin by rat lung cells. Antimicrob. Chemother. 26 (1990) 503 -513 .

${ }^{4}$ Cachet, Th., I. O. Kibwage, E. Roets, J. Hoogmartens, H. Vanderhaeghe: Optimization of the separation of erythromycin and related substances by high-performance liquid chromatography. J. Chromatogr. 409 (1987) 91-100.

5 Neu, H. C.: The development of macrolides: Clarithromycin in perspective. J. Antimicrob. Chemother. 27, Suppl. A (1991) 1 $-9$.

6 Peters, F.: Gutartige Erkrankungen der Brust. Urban und Schwarzenberg, München (1991) 79.
Prof. Dr. med. F. Peters

Frauenklinik St. Hildegardis-Krankenhaus Akademisches Lehrkrankenhaus der Johannes-Gutenberg-Universität Mainz Hildegardstr. 2

55131 Mainz 